\title{
Immediate early response protein 2 regulates hepatocellular carcinoma cell adhesion and motility via integrin $\beta 1$-mediated signaling pathway
}

\author{
ZHENGXIN XU ${ }^{1 *}$, LEI ZHU $^{1 *}$, WENJUAN WU ${ }^{1,2 *}$, YUEXIA LIAO $^{1 *}$, WEICHENG ZHANG $^{1}$, ZIJING DENG $^{1}$, \\ JINGYUAN SHEN $^{1}$, QING YUAN ${ }^{1}$, LU ZHENG $^{1}$, YU ZHANG $^{1}$ and WEIGAN SHEN ${ }^{1,3,4}$ \\ ${ }^{1}$ School of Medicine and ${ }^{2}$ Clinical Medical College, Yangzhou University, Yangzhou, Jiangsu 225001; \\ ${ }^{3}$ Jiangsu Co-Innovation Center for Prevention and Control of Important Animal Infectious Diseases and Zoonoses, \\ Yangzhou, Jiangsu 225009; ${ }^{4}$ Jiangsu Key Laboratory of Integrated Traditional Chinese and \\ Western Medicine for Prevention and Treatment of Senile Diseases, Yangzhou, Jiangsu 225001, P.R. China
}

Received June 4, 2016; Accepted October 25, 2016

DOI: $10.3892 /$ or.2016.5215

\begin{abstract}
Human immediate early response 2 (IER2) has been reported to function as a potential transcriptional factor or transcriptional co-activator and seems to play a pivotal role in tumor cell motility and metastasis, however, its role and underlying mechanisms in hepatocellular carcinoma (HCC) remain unknown. Herein, we demonstrated that overexpression of IER 2 in HCC cells increased cell adhesion to fibronectin, migration and invasion, whereas knockdown of IER2 displayed the opposite effects. In agreement with this phenotype, IER 2 expression was positively correlated with the metastatic potential and integrin $\beta 1$ (ITGB1) expression in HCC cell lines. Moreover, we demonstrated a critical role for IER2 in regulation of HCC cell-extracellular matrix (ECM) adhesion and motility by the transcriptionally promoted ITGB1. Furthermore, we showed that ITGB1-focal adhesion kinase (FAK)-Src-paxillin signal pathway activated by IER2 may contribute to the HCC cell-ECM adhesion and motility. These results demonstrated that IER2 promoted HCC cell adhesion and motility probably by directly increasing ITGB1 expression and subsequently activating the ITGB1-FAK-Src-paxillin signal pathway.
\end{abstract}

\section{Introduction}

Immediate early response 2 (IER2) or ETR101, a human homolog of mouse and rat for pip92 is a cellular immediate

Correspondence to: Professor Weigan Shen or Professor Yu Zhang, School of Medicine, Yangzhou University, 11 Huaihai Road, Yangzhou, Jiangsu 225001, P.R. China

E-mail: shenweigan@hotmail.com

E-mail: yzzy10182001@aliyun.com

*Contributed equally

Key words: immediate early response 2, cell motility, cell adhesion, integrin early response protein that is induced by extracellular stimuli, such as growth factors, 12-O-tetradecanoylphorbol-13-acetate, and some infection of pathogens (1-4). Previous studies have demonstrated that IER2 may function as a fibroblast growth factor intracellular binding protein 1-interacting protein, or as a DNA-binding protein acting as a transcriptional activator for myo-inositol 1-phosphate synthase gene $(3,5)$. Recently, evidence suggests that IER2 seems to play a pivotal role in tumor cell motility and metastasis (6), however, little is known about the mechanism involved. We have previously shown that IER2 regulated human umbilical vein endothelium cell motility, adhesion, in vitro capillary tube formation, and the actin cytoskeleton rearrangement in a FAK-dependent manner (7). Furthermore, we also found that IER2, as a direct and functional target for miR-30c in the hepatocellular carcinoma (HCC) cell lines (SMMC-7721 and HepG2 cells), may function as a positive regulator in cell migration and invasion (8), suggesting that IER2 may play a role in the HCC cell motility.

HCC is the fifth most commonly diagnosed human cancer worldwide, and the leading cause of death for the vast majority of HCC patients is a result of the direct metastases (9), which is a complex process including cancer cells shed from the primary organization, cell-extracellular matrix (ECM) adhesion, cell migration and invading ECM, and each step of metastases is associated with changes in gene expression involved in cell migration, invasion and adhesion $(10,11)$. Thus, understanding the underlying mechanisms involved in these processes will likely contribute to our insight to developing strategies for maximizing the efficacy of HCC treatment. Previous studies have shown that IER2 is upregulated in some metastatic cancer cells and in several human primary tumors, such as pancreas cancer, breast cancer and HCC (6), and IER2 expression promotes tumor cell migration and invasion and correlates with the colorectal adenocarcinoma metastasis $(6,8)$. However, the biological roles and underlying mechanisms involved in HCC of IER 2 are largely unknown.

In the present study, we aimed to investigate the effect of IER 2 on HCC cell motility and cell-ECM adhesion and 
spreading, and to unveil the underlying mechanisms by which IER2 regulated HCC cell motility and adhesion. We demonstrated that overexpression of IER2 in HCC cells promoted cell adhesion to fibronectin and motility, whereas knockdown expression of IER2 displayed the opposite effects. Furthermore, we demonstrated a critical role for IER2 in regulation of HCC cell-ECM adhesion and motility probably by the transcriptionally promoted integrin $\beta 1$ (ITGB1) and then activated ITGB1-focal adhesion kinase (FAK)-Src-paxillin signal pathway.

\section{Materials and methods}

Cell lines and culture conditions. Human embryonic kidney (HEK) $293 \mathrm{~T}$ cells, HCC cell lines HepG2 and SMMC-7721 were obtained from the Type Culture Collection of the Chinese Academy of Sciences (Shanghai, China), and MHCC97H and HCCLM3 cells were obtained from the KeyGen Biotech (Nanjing, China). All cells were routinely maintained in DMEM (Gibco-BRL, Grand Island, NY, USA) supplemented with $10 \%$ fetal bovine serum, $100 \mathrm{U} / \mathrm{ml}$ penicillin and $100 \mu \mathrm{g} / \mathrm{ml}$ streptomycin, and cultured at $37^{\circ} \mathrm{C}$ in a humidified atmosphere of $5 \% \mathrm{CO}_{2}$.

Plasmid construction and lentiviral production. The full-length of human IER2 cDNA was amplified from pEZ-Lv105-IER2 vector obtained from GeneCopoeia (Rockville, MD, USA) by polymerase chain reaction (PCR) using the primers: forward, 5'-ccggaattctggccaccatggaagtgcagaaagaggcac-3', and reverse, 5'-cgcggatcccggaaggccaccacggccegc-3', and followed by cloning into EcoRI and BamHI sites of the pEGFP-N1 vector (Clontech, Mountain View, CA, USA) to generate pEGFP-N1-IER2 vector, which contained a Kozak sequence and ATG to be in frame with GFP on the vector. Cells were transfected with pEGFP-N1-IER2 or pEGFP-N1 vector using Lipofectamine 3000 according to the manufacturer's instruction (Invitrogen), and G418-resistant transfectants were selected. Construct expressing shRNA specific targeting ITGB1 (shITGB1) was produced by annealing the synthesized primers, 5'-CCGGCAAGAGAGC TGAAGACTATCTCGAGATAGTCTTCAGCTCTCTTGTTT TTG-3', sense and 5'-AATTCAAAAACAAGAGAGCTGAA GACTATCTCGAGATAGTCTTCAGCTCTCTTG-3', antisense and cloned into the AgeI and EcoRI sites of pGV115, and the non-silencing control shRNA vector (NC) were both obtain from the GeneChem Corporation (Shanghai, China). Cells were transfected with shITGB1 or with NC using Lipofectamine 3000 according to the manufacturer's instruction. ITGB1 promoter was cloned from human genomic DNA by PCR using the primers: forward, 5'-CGGGGTACCGATAGCAGCTTGCCA GTAGC-3' and reverse, 5'-CCCAAGCTTGGTGGCGGCGG CTCCTCCTCCT-3', and then inserted into the pGL3-basic vector (Promega) at KpnI and HindIII sites. All of the constructs were validated by DNA sequencing.

The production and transduction of the recombinant lentiviruses encoding IER2 (LV-IER2), shRNA against human IER2 (LV-shR), and indicated control lentiviruses (LV-C and LV-shC) were generated or acquired as previously described $(7,8)$. After lentiviral-infection for $48 \mathrm{~h}$, the growth medium was supplemented with puromycin and maintained at least for two weeks to select stably transduced cell lines, and cells were screened for IER 2 expression by real-time reverse transcription quantitative PCR (RT-qPCR) and western blotting.

$R T-q P C R$. Total RNA was extracted and purified from the cells using the RNA isolator total RNA extraction reagent, and subjected to reverse transcription using the HiScript First Strand cDNA synthesis kit according to the supplier's instruction (Vazyme, Nanjing, China). The real-time PCR and data collection were subsequently performed as described previously using the AceQ ${ }^{\circledR}$ qPCR SYBR ${ }^{\circledR}$ Green master mix kit (Vazyme) (12). Primers used for the amplification were as follows: IER2 forward, 5'-CCAAAGTCAGCCGCAAACGA-3' and reverse, 5'-TTT CTTCCAGACGGGCTTTCTTGC-3'; ITGB1 forward, 5'-AAT GTAACCAACCGTAGC-3' and reverse, 5'-CAGGTCCATAAG GTAGTAGA-3'; integrin $\alpha 5$ (ITGA5) forward, 5'-AGGCAGCT ATGGCGTCCCACTGT-3' and reverse, 5'-CATCAGAGGTG GCTGGAGGCTTG-3'; and glyceraldehyde-3-phosphate dehydrogenase (GAPDH) forward, 5'-GCACCGTCAAGGCTGAG AAC-3' and reverse, 5'-TGGTGAAGACGCCAGTGGA-3'. The relative expression levels of the indicated mRNA normalized against GAPDH mRNA were calculated using the $2^{-\Delta \Delta \mathrm{CT}}$ methods.

Western blot analysis. Western blot analysis was performed following standard methods. Briefly, cell lysates were prepared, separated and transferred onto polyvinylidene fluoride membranes (Millipore, Billerica, MA, USA). The membranes were then blocked with $5 \%$ nonfat dried milk or $3 \%$ bovine serum albumin (BSA) for tyrosine phosphorylation blots and incubated with primary antibodies, and followed by incubation with horseradish peroxidase (HRP)-linked secondary antibodies. The antibodies used in the current study were: mouse polyclonal anti-IER2 (1:1,000; Abcam); rabbit monoclonal anti-ITGB1, rabbit polyclonal anti-ITGA5, rabbit monoclonal anti-FAK, rabbit polyclonal anti-pY397FAK, rabbit polyclonal anti-pY576/Y577FAK, rabbit polyclonal anti-pY925FAK, rabbit monoclonal anti-Src, rabbit polyclonal anti-pY527Src (1:1,000), HRP-linked anti-mouse IgG and HRP-linked anti-rabbit IgG (1:2,000) (Cell Signaling Technology, Danvers, MA, USA); goat polyclonal anti-pY407FAK, goat polyclonal anti-pY861FAK, goat polyclonal anti-pS910FAK, mouse monoclonal anti-paxillin and goat polyclonal anti-pY118paxillin (1:200; Santa Cruz Biotechnology, Inc., Santa Cruz, CA, USA); and mouse monoclonal anti-GAPDH $(1: 1,000)$ and HRP-linked donkey anti-goat IgG $(1: 3,000)$ (KangChen Biotech, Shanghai, China). Immunoreactive protein bands were visualized with the Pierce ECL Plus Western Blotting Substrate (Thermo Fisher Scientific, Rockford, IL, USA). GAPDH was performed as an internal loading control, and quantification of the band intensity was performed using ImageJ software (NIH).

Cell viability and proliferation assay. Cell viability and proliferation was assessed by using the Cell Counting kit- 8 (CCK-8; Obio Technology, Shanghai, China) assay according to the manufacturer's instruction. In brief, aliquots $(100 \mu \mathrm{l})$ of cell suspension of SMMC-7721 or MHCC-97H cells $\left(5 \times 10^{3}\right.$ cells/well) were seeded in 96-well culture plates and cultured in DMEM complete medium for 24, 48 and $72 \mathrm{~h}$. At the indicated time-points, $10 \mu \mathrm{l}$ of CCK- 8 solution was added to each well and incubated for another $1 \mathrm{~h}$ at $37^{\circ} \mathrm{C}$, and the 
absorbance at $450 \mathrm{~nm}$ was measured with a multifunctional microplate reader. Assays were performed in six wells and repeated five times.

Cell migration and invasion assay. Cell migration and invasion assays were performed as described previously (12). In brief, cells were serum-starved overnight and then $200 \mu \mathrm{l}$ of $1 \times 10^{5}$ or $2 \times 10^{5}$ cells in serum free medium were seeded in uncoated or Matrigel-coated chambers with $8.0 \mu \mathrm{m}$ pore sizes (6.5 mm; Costar, Cambridge, MA, USA) of the 24-well Transwell, respectively. The inserts were then placed into the lower chambers containing $600 \mu \mathrm{l}$ of the complete growth medium. The migrated or invaded cells on the lower surface of the inserts were fixed and stained. Five random fields at x100 magnification were analyzed under a light microscope. Assays were performed in triplicate from three independent experiments.

Cell-ECM adhesion and cell spreading assays. Cell-ECM adhesion assays were performed on the collagen type I, fibronectin and Matrigel (BD Biosciences, Franklin Lakes, NJ, USA), respectively. For collagen type I or fibronectin coated, the 96-well culture plates were coated with $10 \mu \mathrm{g} / \mathrm{ml}$ collagen type I or fibronectin overnight at $4^{\circ} \mathrm{C}$ and blocked with $1 \% \mathrm{BSA}$ in PBS at $37^{\circ} \mathrm{C}$ for at least $1 \mathrm{~h}$, and then washed with PBS. For Matrigel coated, Matrigel $(200 \mu \mathrm{g} / \mathrm{ml})$ was added to the 96-well culture plates, incubated at $37^{\circ} \mathrm{C}$ for at least $1 \mathrm{~h}$, and blocked with $1 \%$ BSA. For cell-ECM adhesion assays, aliquots $(100 \mu \mathrm{l})$ of cell suspension at a density of $2 \times 10^{6}$ cells/ $\mathrm{ml}$ were seeded to each well of the matrix coated 96 -well culture plates and allowed to adhere to the matrix for $45 \mathrm{~min}$ at $37^{\circ} \mathrm{C}$. Unattached cells were gently removed and adhesive cells were quantified by using the CCK- 8 assay according to the supplier's instruction. Assays were performed in six wells from five independent experiments. For cell spreading assays, cells ( $2 \times 10^{5}$ cells/well) were seeded to each well of the fibronectin coated coverslips in 24-well culture plates prepared as above and incubated at $37^{\circ} \mathrm{C}$ for the indicated time. The cells were then fixed immediately with $4 \%$ paraformaldehyde and the spreading cells were captured by light microscopy equipped with a digital image capturing system. Spreading cells were defined as cells adherent with protrusions or lamellipodia (fried egg shape), whereas unspreading cells were identified by their round and adherent with less projections appearance. Spreading was quantified from 10 randomly selected fields from three independent experiments.

Chromatin immunoprecipitation (ChIP) assay. The ChIP assay was performed in the cells stably transfected with pEGFP-N1-IER2 or pEGFP-N1 vectors using the SimpleChIP ${ }^{\circledR}$ Plus Enzymatic Chromatin IP kit (Cell Signaling Technology) according to the supplier's instruction. In brief, cells were grown to confluence in culture dishes and fixed in formaldehyde (1\%), scraped from the dishes and lysed, and the homogenized chromatin was sheared by micrococcal nuclease for $20 \mathrm{~min}$ at $37^{\circ} \mathrm{C}$ and sonicated with several pulses to break the nuclear membrane. The GFP antibody (Invitrogen), positive control histone $\mathrm{H} 3$ antibody, or negative control normal rabbit $\mathrm{IgG}$ was used to precipitate the precleared cross-linked chromatin at $4^{\circ} \mathrm{C}$ overnight with rotation, and followed by adding protein $\mathrm{G}$ beads and incubation for $4 \mathrm{~h}$ at $4^{\circ} \mathrm{C}$ with rotation. After washing, the immunoprecipitated chromatin was eluted from the antibody/protein $\mathrm{G}$ beads and purified by reversal of cross-links, removal of RNA with RNase A, and treatment with proteinase $\mathrm{K}$. The immunoprecipitated DNA fragments were performed as the template for PCR using the human ITGB1 promoter-specific primers forward, 5'-TCTTAGACACAGGCAAGCACA-3' and reverse, 5'-CCTGAGTCCCGAGGCAAATC-3'. The PCR products were run on a $2 \%$ agarose gel and visualized with ethidium bromide staining.

Luciferase reporter gene assay. For luciferase reporter gene assay, the luciferase reporter constructs (200 ng) and pTK-Renilla vectors (100 ng) were transiently transfected into the lentiviral-transduced cells in 24-well culture plates using Lipofectamine 3000. After incubation for $48 \mathrm{~h}$, luciferase activities were determined using the luciferase reporter assay system (Promega), and normalized to Renilla luciferase activities.

Statistical analysis. Data are shown as the mean \pm standard deviation (SD) and analyzed by the Student's t-test. The statistical significance was determined by $\mathrm{p}<0.05$.

\section{Results}

IER2 promoted HCC cell migration and invasion. To understand the contribution of IER2 expression to the biological functions in HCC cells, the lentivirus-mediated IER2 overexpression or knockdown in the low metastatic potential SMMC7721 cells and in the high metastatic potential MHCC97H cells were initially established. Overexpression and knockdown of IER2 in SMMC-7721 cells and in MHCC97H cells were confirmed by RT-qPCR (Figs. 1A and 2A) and western blot analysis (Figs. $1 \mathrm{~B}$ and $\mathrm{C}$ and $2 \mathrm{~B}$ and $\mathrm{C}$ ). The data suggested that the indicated lentiviruses have been successfully transduced into the SMMC-7721 and MHCC97H cells, and IER2 overexpression was shown in LV-IER2-infected cells, and efficient knockdown of IER 2 was shown in LV-shR-infected cells, thus, the indicated lentiviruses could be used in the following experiments.

We employed lentivirus-mediated IER2 overexpression or knockdown in the SMMC7721 cells and MHCC97H cells, respectively, and performed Transwell cell migration and invasion assays. Compared with the corresponding empty vector-transduced cells ( $\mathrm{LV}-\mathrm{C}$ and $\mathrm{LV}$-shC) and the nontransduced cells [wild-type (WT)], both SMMC-7721 and MHCC97H cells stably transduced with LV-IER2 showed significant increase of the cell migratory and invasiveness capacity, whereas silencing of IER2 obviously attenuated the motility of SMMC-7721 and MHCC97H cells (Fig. 3A-D). No significant differences in cell motility were observed among the empty vector-infected cells and the WT. In addition, the alteration in cell motility did not appear to be due to affected cell viability and proliferation. Under these conditions, knockdown and the ectopic expression of IER2 had no obvious effects on cell viability and proliferation of SMMC-7721 and MHCC97H cells in vitro (Fig. 3E and F). These observations supported the notion that IER2 played an important role in HCC cell migration and invasion. 

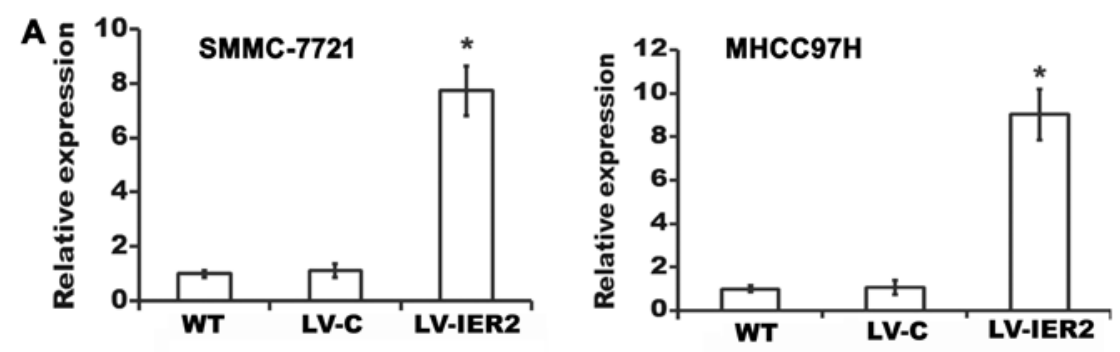

$\mathbf{B}$

SMMC-7721

MHCC97H
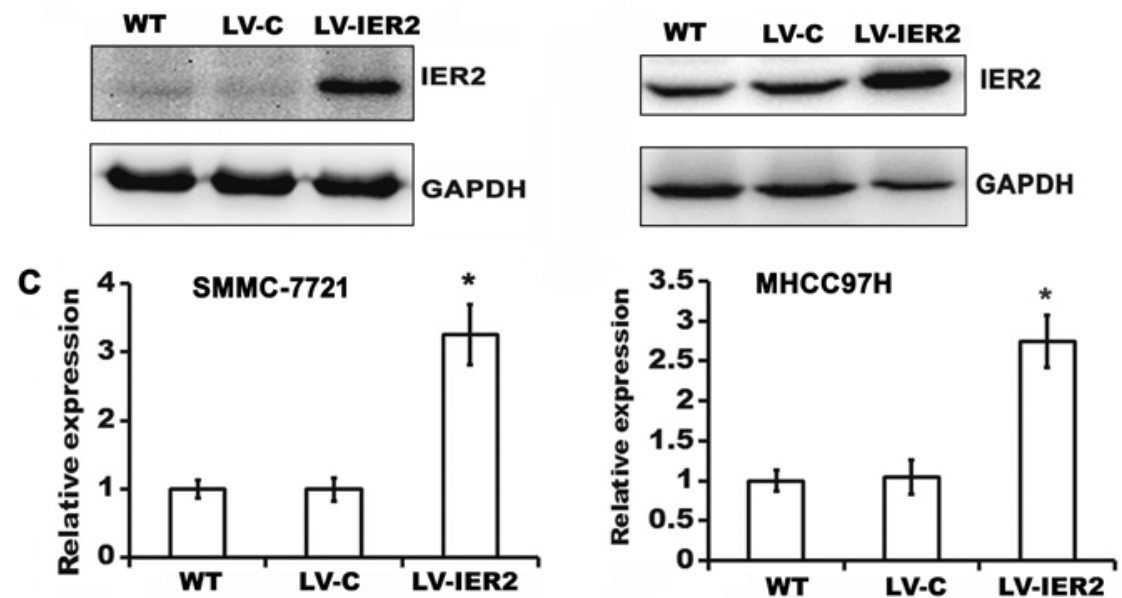

Figure 1. Overexpression of IER2 in the lentiviral-transduced SMMC-7721 and MHCC97H cells. (A) IER2 mRNA levels were quantified from the indicated lentiviral-transduced SMMC-7721 and MHCC97H cells using RT-qPCR, and normalized against GAPDH. Data are the mean \pm SD ( $n=5$ ). " $p<0.05$ vs. the WT. (B and C) Overexpression of IER2 protein was determined by western blotting, normalized to GAPDH. The values are the mean \pm SD ( $n=3$ ). " $p<0.05$ vs. the WT.

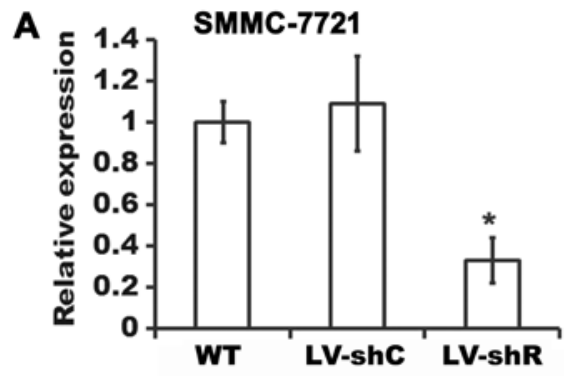

SMMC-7721

B

SMMC-7721

WT LV-shC LV-shR
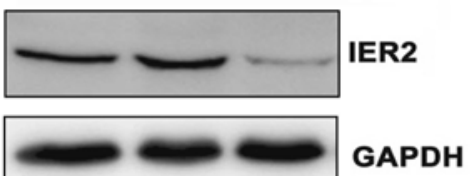

C

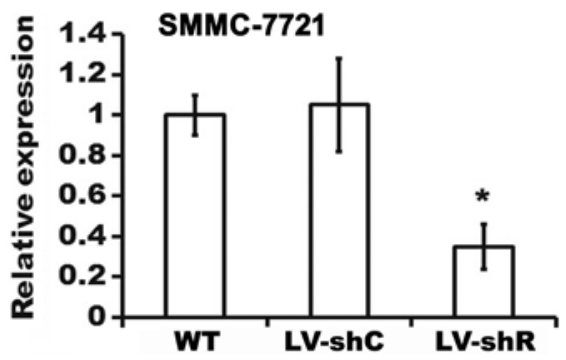

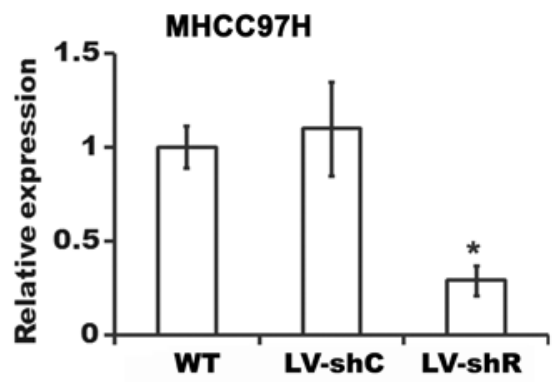

МHCC97H
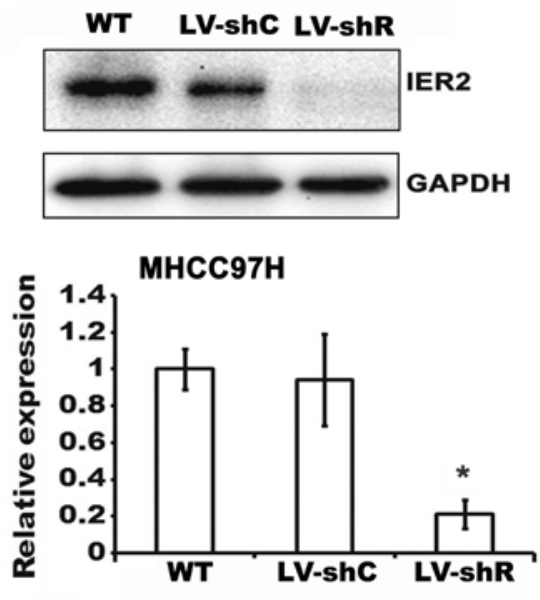

Figure 2. Knockdown of IER2 in the lentiviral-transduced SMMC-7721 and MHCC97H cells. (A) IER2 mRNA levels were evaluated from the indicated lentiviral-transduced SMMC-7721 and MHCC $97 \mathrm{H}$ cells using RT-qPCR, and normalized to GAPDH. Data are the mean \pm SD $(n=5)$. " $p<0.05$ vs. the WT. (B and C) Knockdown of IER2 protein was determined by western blotting, normalized to GAPDH. The values are the mean \pm SD ( $n=3$ ). ${ }^{*} p<0.05$ vs. the WT. 

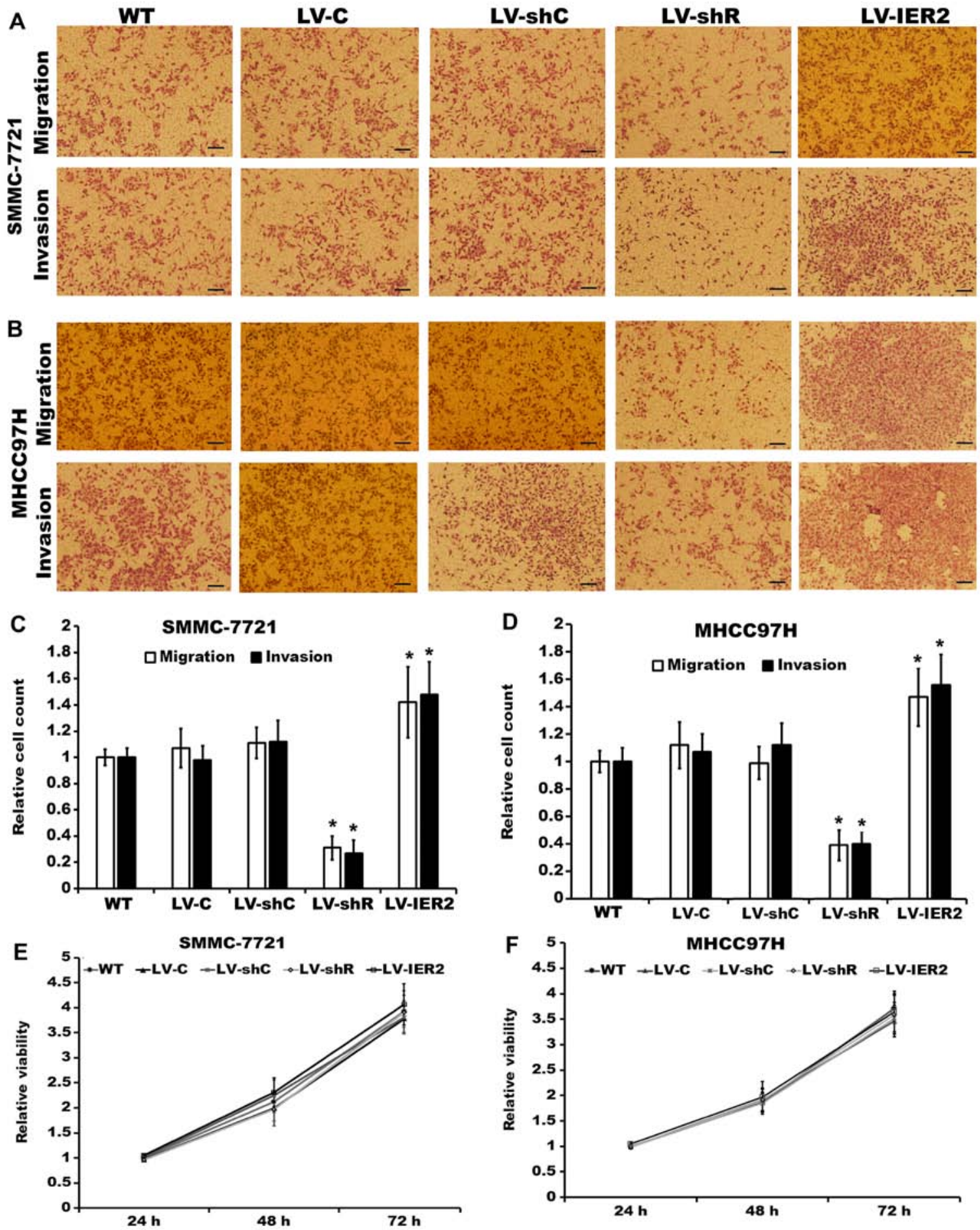

Figure 3. Effect of IER 2 on cell migration and invasion. (A and B) Representative Transwell cell migration and invasion assays in the indicated lentiviral-transduced (A) SMMC-7721 and (B) MHCC97H cells. Scale bars, $100 \mu \mathrm{m}$. (C and D) Quantification of the migration and invasion of the lentiviral-transduced (C) SMMC-7721 and (D) MHCC97H cells is shown. Assays were performed in triplicate from three independent experiments and data are the mean \pm SD. " $\mathrm{p}<0.05$ vs. the WT. (E and F) Quantification of the relative cell proliferation of the lentiviral-transduced (E) SMMC-7721 and (F) MHCC97H cells were shown. Values are the mean \pm SD of five independent experiments.

IER2 regulates cell-ECM adhesion and spreading. Considering that cell adhesion and spreading onto ECM are crucial steps in cellular migration and invasion (13), we then investigated whether IER2 positively regulates HCC cell-ECM adhesion and spreading. Lentiviral-transduced SMMC-7721 or MHCC97H cells were seeded on collagen type I-, Matrigel- or fibronectin-coated 96-well culture plates, respectively, and incubated at $37^{\circ} \mathrm{C}$ for $45 \mathrm{~min}$. As shown in Fig. 4, compared with those in the empty vector-transduced cells and WT, LV-IER2 infection significantly promoted SMMC-7721 or MHCC97H cell adhesion onto the fibronectin, while LV-shR infection decreased the cell adhesion onto the fibronectin (Fig. 4C). Interestingly, there were no significant alteration in the cell adhesion onto the collagen type I or Matrigel either in IER2 overexpressing cells or IER2 knockdown cells (Fig. 4A and B). No significant difference was observed either in the LV-C-infected or the LV-shC-infected 

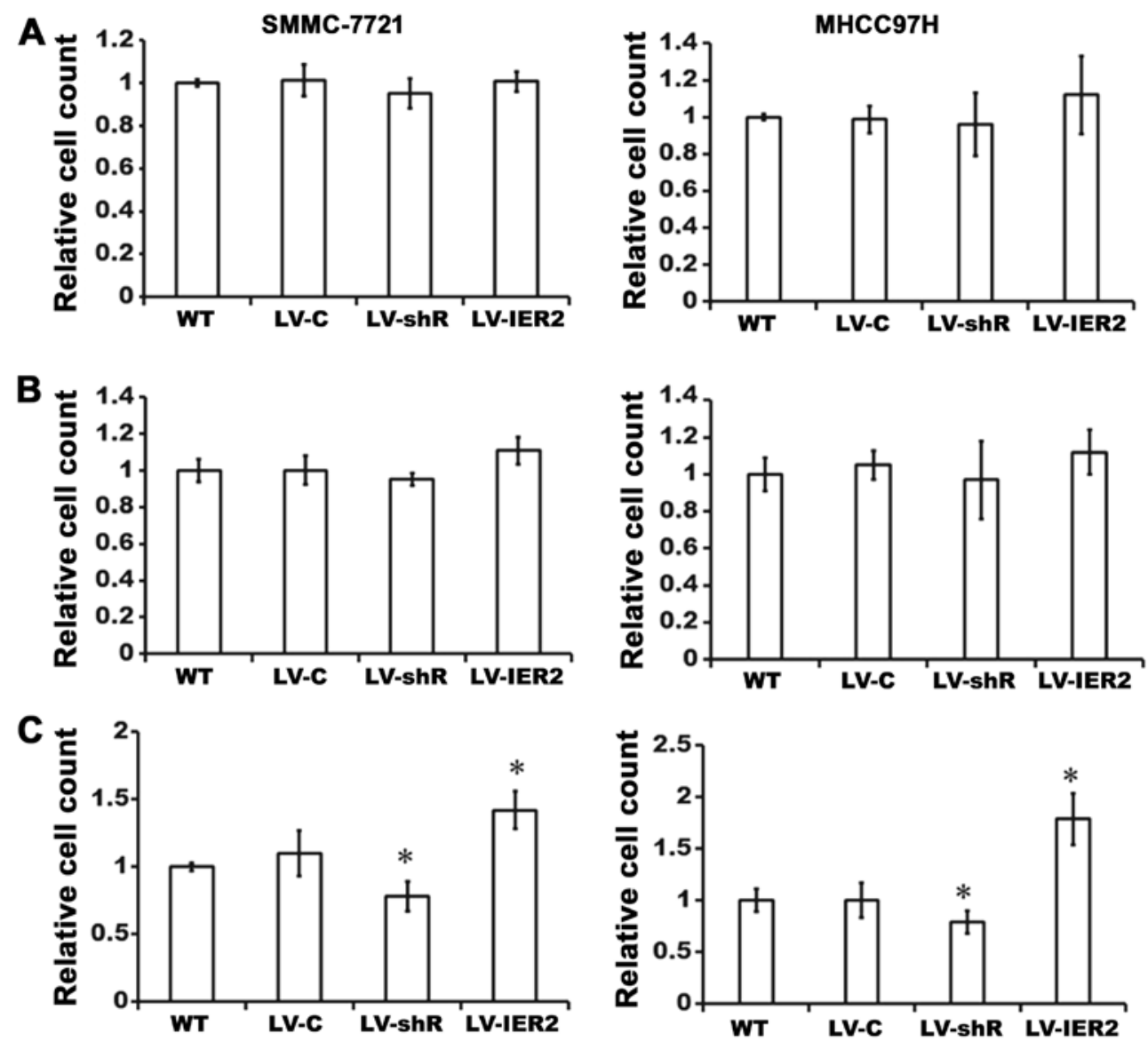

Figure 4. Effect of IER2 on cell-ECM adhesion. The lentiviral-transduced SMMC-7721 (left) and MHCC97H cells (right) were seeded onto the 96-well culture plates pre-coated with (A) collagen type I, (B) Matrigel, or (C) fibronectin, respectively, and incubated at $37^{\circ} \mathrm{C}$ for 45 min. The number of the adhesive cells was quantified using the CCK-8 assays by absorbance at $450 \mathrm{~nm}$. Assays were performed in six wells from five independent experiments and data are the mean \pm SD. " $p<0.05$ vs. the WT.

cells (data not shown). These data demonstrated that IER2 is an important regulator of $\mathrm{HCC}$ cell adhesion onto the fibronectin, but not collagen type I or Matrigel, and promotion of the cell motility by IER 2 overexpression may be the consequence of the increase cell-fibronectin adhesion, while the reduction in the cell motility induced by IER 2 knockdown is likely the consequence of the decreasing cell-fibronectin adhesion.

Since cell spreading is the early phase of cell migration and invasion $(13,14)$, and IER2 expression promoted cell adhesion onto the fibronectin (but not collagen type I or Matrigel) as described above, we further examined whether IER2 positively regulated the cell spreading on the fibronectin. With increasing the time of incubation, both SMMC-7721 and MHCC97H cells showed significant increase of the cell spreading (Fig. 5). Specifically, when compared to empty vector-transduced cells or WT, overexpression of IER2 significantly increased the ability of cells to spread on the fibronectin coated surface (Fig. 5C and D), and the LV-IER2-transduced cells displayed increased cell spreading surface area and showed lamellopodia around their peripheral edges (Fig. 5A and B). On the contrary, IER2 depletion obviously decreased cell spreading on the fibronectin, and these IER2 silencing cells showed poor spreading and exhibited a non-polarized, spherical shape, and few lamellipodi protrusions (Fig. 5A and B). No significant difference was found either in the LV-C-transduced or the LV-shC-transduced cells (data not shown). Taken together, these results suggested that IER2 is required for effective spreading and adhesion of HCC cells to fibronectin, and that the increased cell adhesion and spreading of IER 2 expressing cells, perhaps account for the promotion of IER2 on migration and invasion.

IER 2 promotes ITGB1 expression in HCC cells. ITGA5 and ITGB1 form heterodimers to mediate cell adhesion onto the fibronectin (15) and play very important roles in cell motility (16-18). Since IER2 expression positively regulated cell motility, adhesion and spreading onto the fibronectin of the SMMC-7721 and MHCC97H cells (Figs. 3-5), we then tested whether IER 2 expression was correlated with the metastatic potential of HCC cell lines and with ITGA5 or ITGB1 expression both in mRNA and protein levels in HCC cells. Data from RT-qPCR and western blot analysis demonstrated that the HCC cell lines, HepG2, SMMC-7721, MHCC97H and HCCLM3 cells, four HCC cell lines with increasing spontaneous metastatic potential (19-21), were shown to express IER2 with relative low expression of IER2 in HepG2 and SMMC-7721 cells, which have low metastatic potential, and abundant expression of IER2 in MHCC97H and HCCLM3 cells, which have high metastatic potential (Fig. 6), indicating that IER2 expression was positively correlated with the metastatic potential of HCC cell lines. Moreover, the results showed that IER2 expression was positively correlated with ITGB1 
A
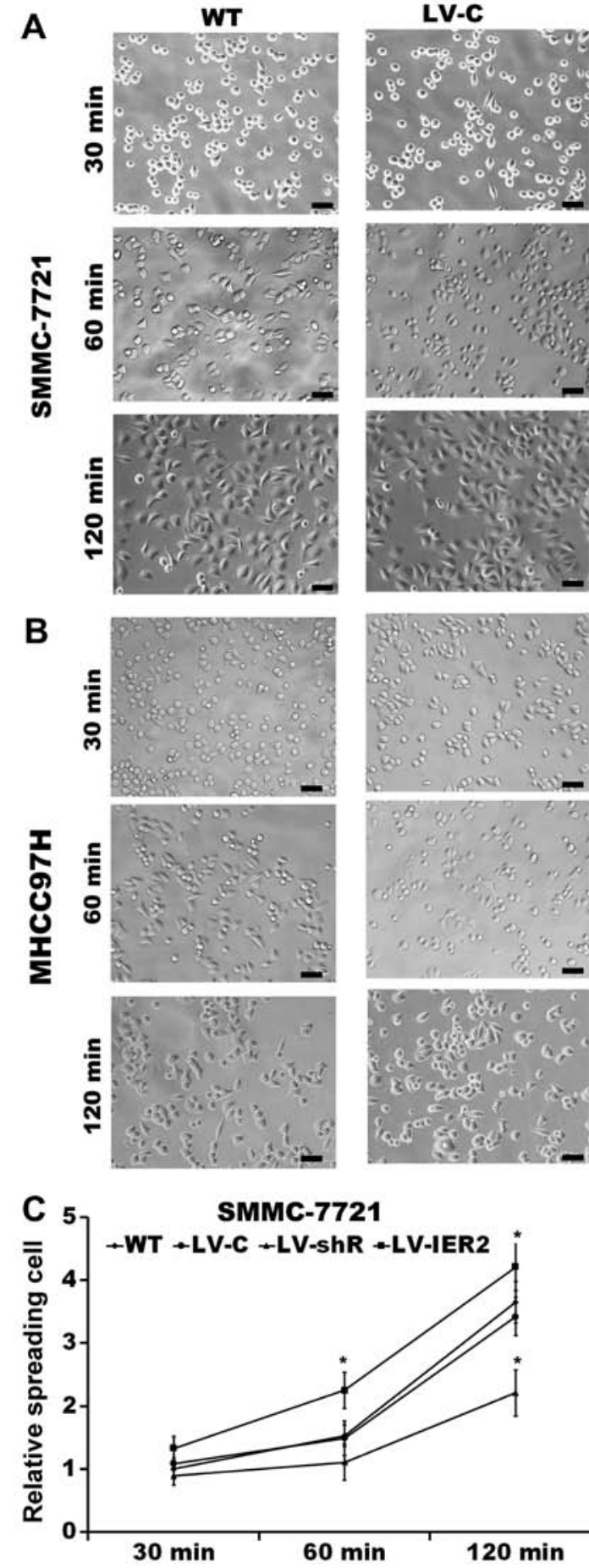

LV-C
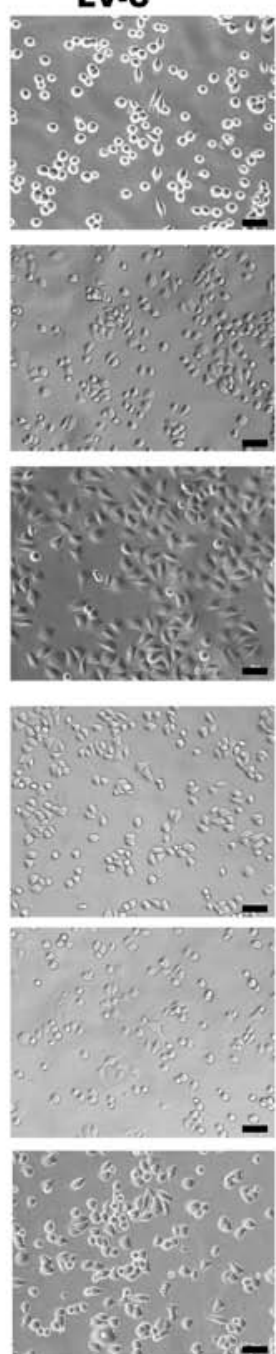
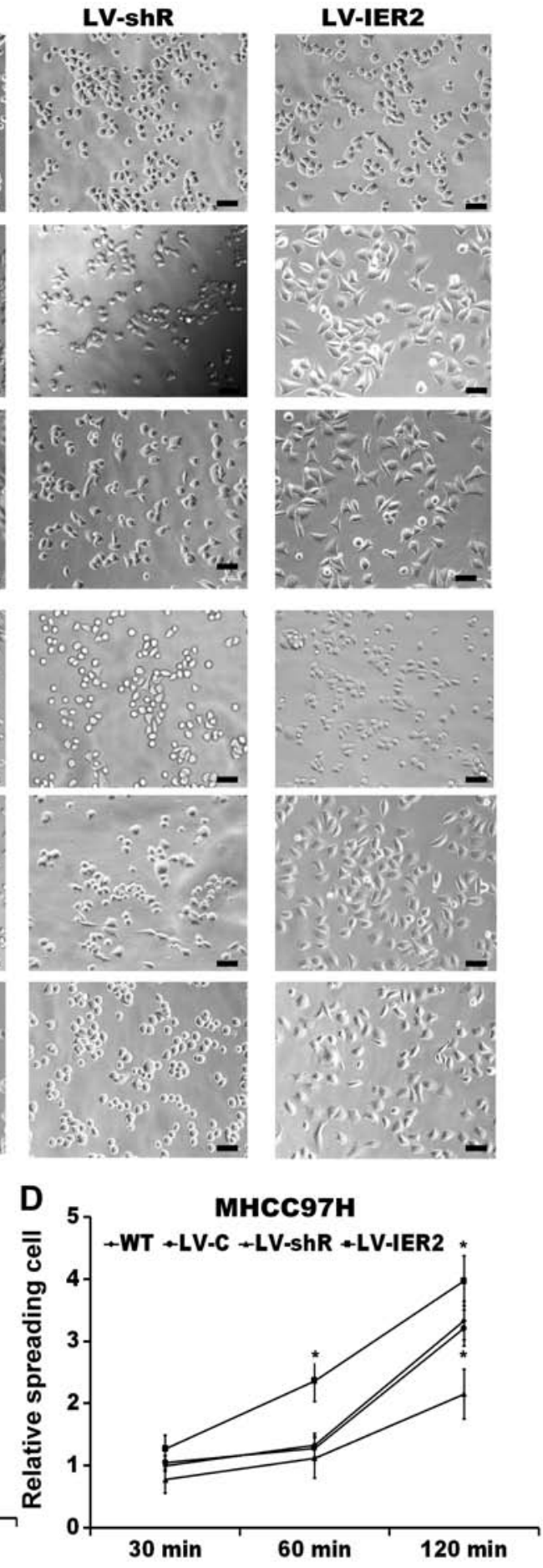

Figure 5. Effect of IER2 on cell spreading to the fibronectin. (A and B) Indicated lentiviral-transduced (A) SMMC-7721 and (B) MHCC97H cells were seeded to each well of the fibronectin-coated coverslips in 24 -well plates and subjected to cell spreading assay at $37^{\circ} \mathrm{C}$ for the indicated time. The images represent the cells from three independent experiments. Scale bars, $100 \mu \mathrm{m}$. (C and D) Quantification of relative cell spreading at the indicated time after seeding on fibronectin. Data are presented as mean \pm SD of three independent experiments. ${ }^{*}$ p $<0.05$ vs. the WT.

(but not ITGA5) expression in these cell lines with increasing spontaneous metastatic potential (Fig. 6).

We further explored whether IER2 expression may regulate ITGA5 or ITGB1 expression both in mRNA and protein levels. Our results indicated that abundant expression of ITGA5 was found in HCC cell lines (Figs. 6B and 7C and D), whereas IER2 overexpression or knockdown resulted in significant upregulation or downregulation of ITGB1, but not ITGA5 either in mRNA or protein levels in SMMC-7721

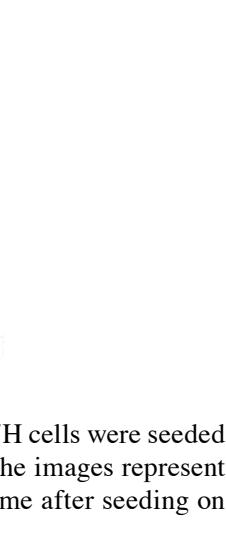

and MHCC97H cells (Fig. 7A-F), suggesting that IER2 expression levels positively correlate with ITGB1 expression in SMMC-7721 and MHCC97H cells. To verify whether the observed positive correlation of IER2 with the ITGB1 expression is the consequence of the interaction of IER2 with the ITGB1 promoter, the luciferase reporter gene assay and ChIP assay were performed. After transient transfection of the luciferase reporter constructs and the internal control vectors into the indicated lentiviral-transduced SMMC-7721 and 

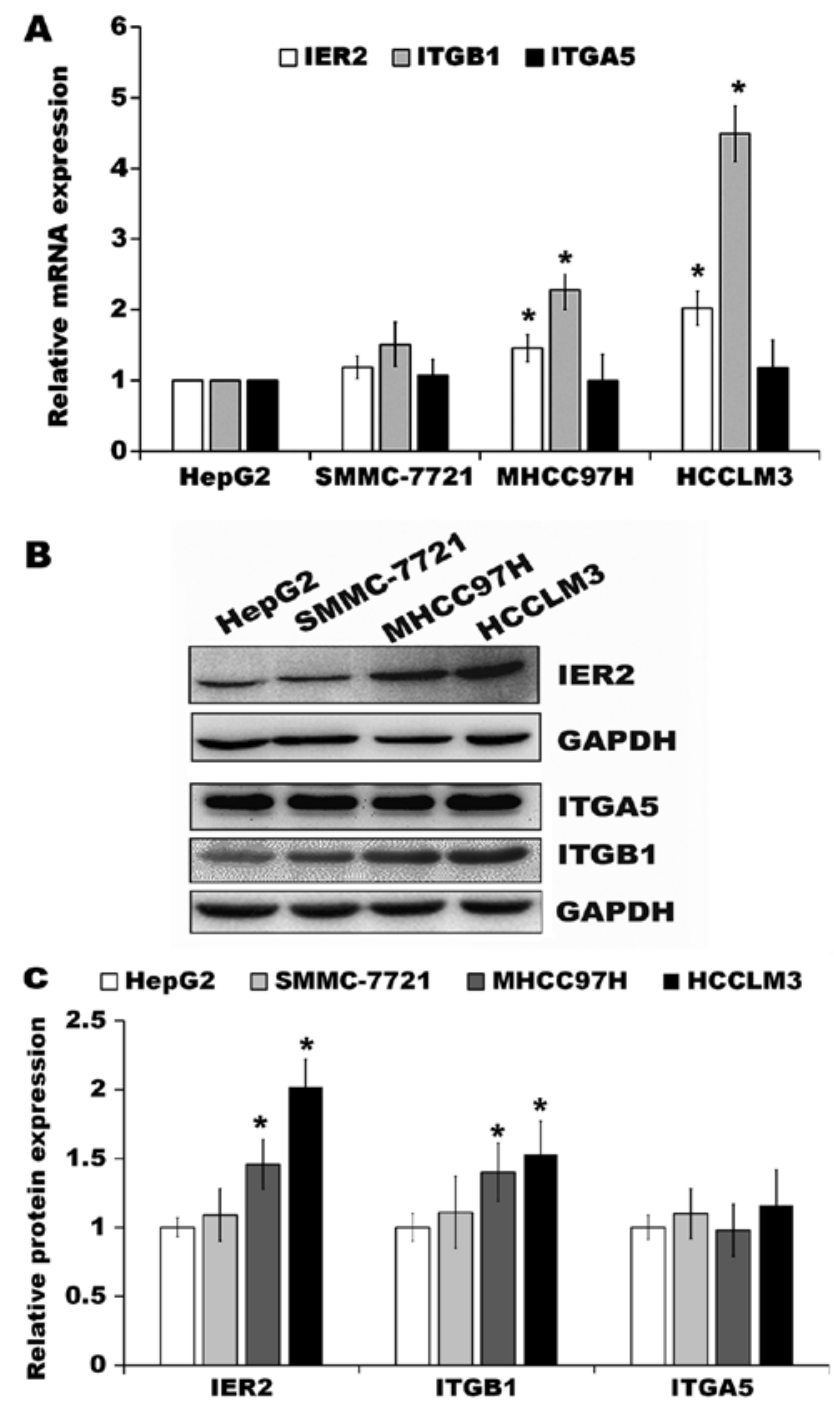

Figure 6. Expression of IER2, ITGA5 and ITGB1 in HCC cells. (A) Levels of IER2, ITGA5 and ITGB1 mRNA were determined by RT-qPCR in RNA extracts from HepG2, SMMC-7721, MHCC97H and HCCLM3 cells, respectively, and normalized to GAPDH. Data are the mean $\pm \mathrm{SD}(\mathrm{n}=5)$. "p $<0.05$ vs. the HepG2 cells. (B and C) Protein levels of IER2, ITGA5 and ITGB1 were measured in HCC cells by western blot assay, and normalized to GAPDH $(n=3)$. The values are the mean $\pm \operatorname{SD}(n=3)$. ${ }^{*} p<0.05$ vs. the WT.

MHCC 97H cells, overexpression of IER2 markedly upregulated the luciferase activity of the ITGB1 promoter, while knockdown of IER2 significantly decreased the luciferase activity of the ITGB1 promoter in comparison with those in the WT and empty vector-transduced cells (Fig. 7G and H). Moreover, results from ChIP assay, which was performed in SMMC-7721 and MHCC97H cells stably transfected with pEGFP-N1-IER2 or pEGFP-N1 vectors, showed that the amplified product of $146 \mathrm{bp}$ was enriched in the pEGFP-N1-IER2 expressing cells relative to that in the negative control IgG and the input (Fig. 7I and J), indicating that IER2 does interact directly with the ITGB1 promoter. Collectively, these data demonstrated that IER2 may regulate expression of the ITGB1 in HCC cells by direct interaction with the ITGB1 promoter, and indicated that IER 2 promoted the cell-fibronectin adhesion and motility of the HCC cells presumably by activating ITGB1 signaling.
Effect of IER 2 on the ITGB1-mediated signaling pathway. In present study we observed that IER 2 promoted cell adhesion and spreading on the fibronectin (Figs. 4 and 5) and regulated expression of the ITGB1 (Fig. 7) in HCC cell lines, suggesting that IER2-mediated ITGB1 expression may account for the promotion of cell motility by activating ITGB1-mediated signaling pathway. To assess the requirement of ITGB1 in IER2-promoted cell spreading and motility, ITGB1 was knocked down in both SMMC-7721 and MHCC97H cells stably transduced with LV-C or LV-IER2, and these cells were subjected to cell spreading, Transwell migration and invasion assays. As shown in Fig. 8,compared with thosein the cellstransfected with non-targeting shRNA (NC), ITGB1 knockdown in stably LV-C-transduced SMMC-7721 (SMMC-7721-LV-C) and MHCC97H (MHCC97H-LV-C) cells or the LV-IER2transduced SMMC-7721 (SMMC-7721-LV-IER2) and MHCC97H (MHCC97H-LV-IER2) cells significantly reduced cell spreading, migration and invasion, suggesting that the elevated ITGB1 resulted from IER2 overexpression in the SMMC-7721 or MHCC97H cells plays an important role in promoting cell motility.

It has been well characterized for most cell types that integrins link ECM to actin filaments activating the integrin-mediated downstream signaling molecules, including FAK, Src and paxillin, thereby regulating the cell-ECM adhesion, spreading and cell motility (16-18,22-24). We next performed western blot assay to explore whether IER2 expression regulated cell motility by activating FAK-Src-paxillin signaling pathway. As shown in Fig. 9, compared with those in the WT and empty vector-transduced cells, overexpression of IER2 significantly increased phosphorylation of FAK at Y397 (pY397FAK), Y407 (pY407FAK), Y576/Y577 (pY576/ Y577FAK), Y861 (pY861 FAK), Y925 (pY925FAK) and S910 (pS910FAK), and phosphorylation of Src at Y419 (pY419Src) and paxillin at Y118 (pY118paxillin), and decreased phosphorylation of Src at Y527 (pY527Src). Interestingly, knockdown of IER2 showed a slight, but not significant alteration of phosphorylation of FAK and paxillin, whereas, obvious decrease of pY527Src and increase of pY419Src were shown in IER2 silencing cells, suggesting that IER2 activated ITGB1-mediated signal pathway is likely a part of a mechanism that participates in modulation of cell motility and adhesion of HCC cells. No significant differences were found either in the LV-C-transduced or the LV-shC-transduced cells (data not shown). Taken together, these results indicated that IER2-mediated ITGB1 expression is partially responsible for IER2-promoted cell-fibronectin adhesion and motility of the HCC cells, providing a novel molecular mechanism for IER2 promoting cell adhesion and motility.

\section{Discussion}

Previously, a study showed that IER 2 plays a role in tumor cell motility and metastasis (6), and we also found that IER2 promotes the motility of SMMC-7721 and HepG2 cells (8). However, the detailed roles and underlying molecular mechanisms in HCC remained unknown. In this study, we investigated the effects of IER2 on the migration, invasion, cell-ECM adhesion and spreading of two HCC cell lines SMMC-7721 with low metastatic potential and MHCC97H 
A

SMMC-7721

QWT QLV-C םLV-shR QLV-IER2

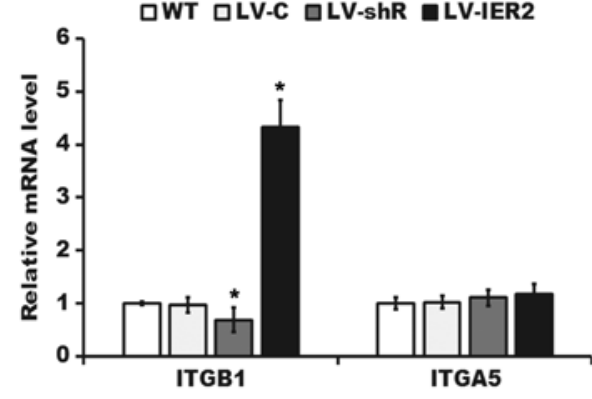

C

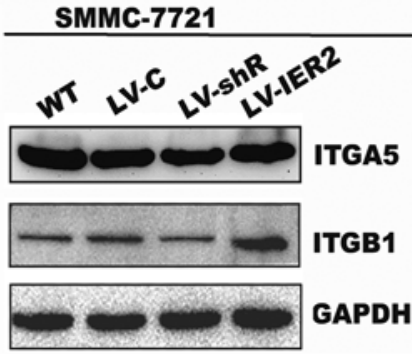

E
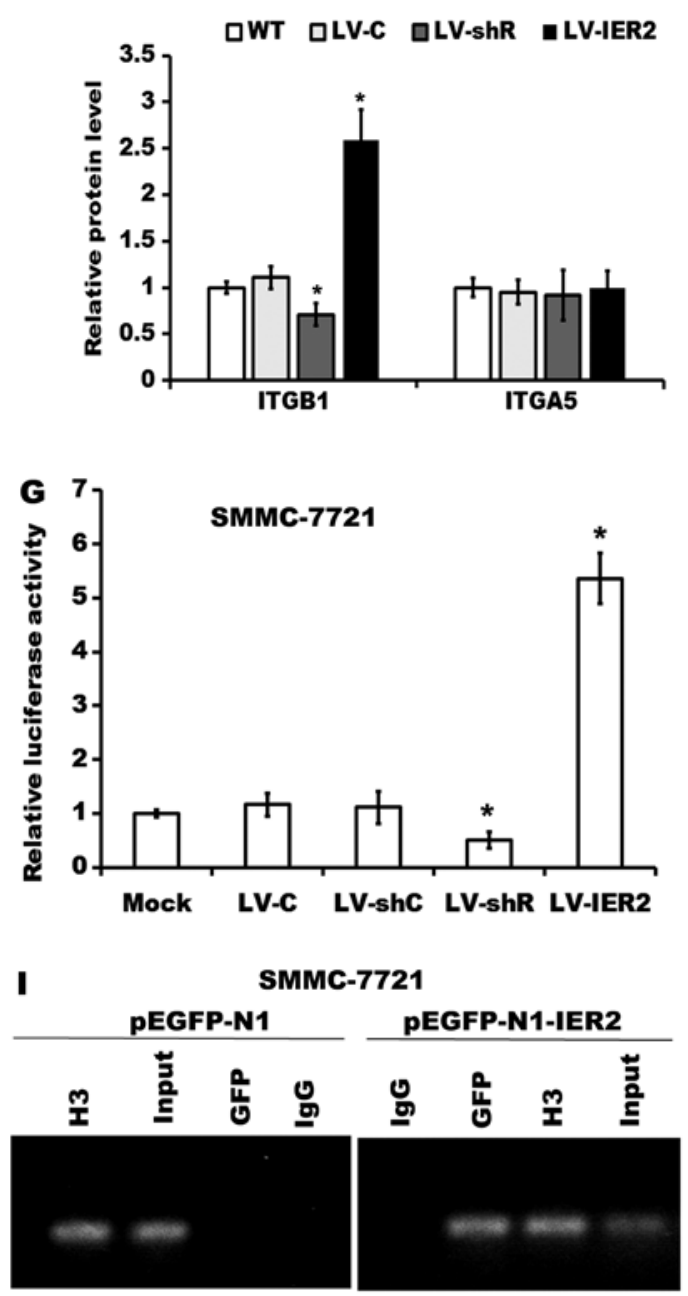

B

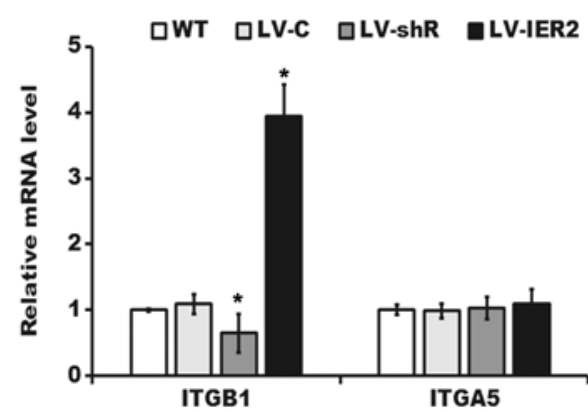

D

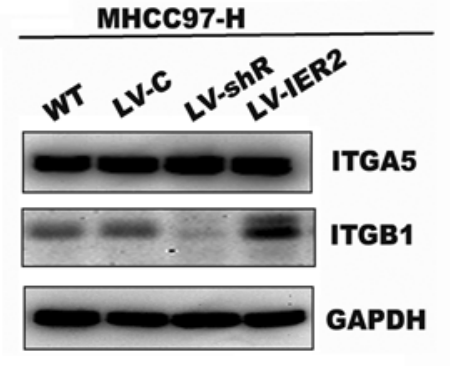

$\boldsymbol{F}$

MHCC97H
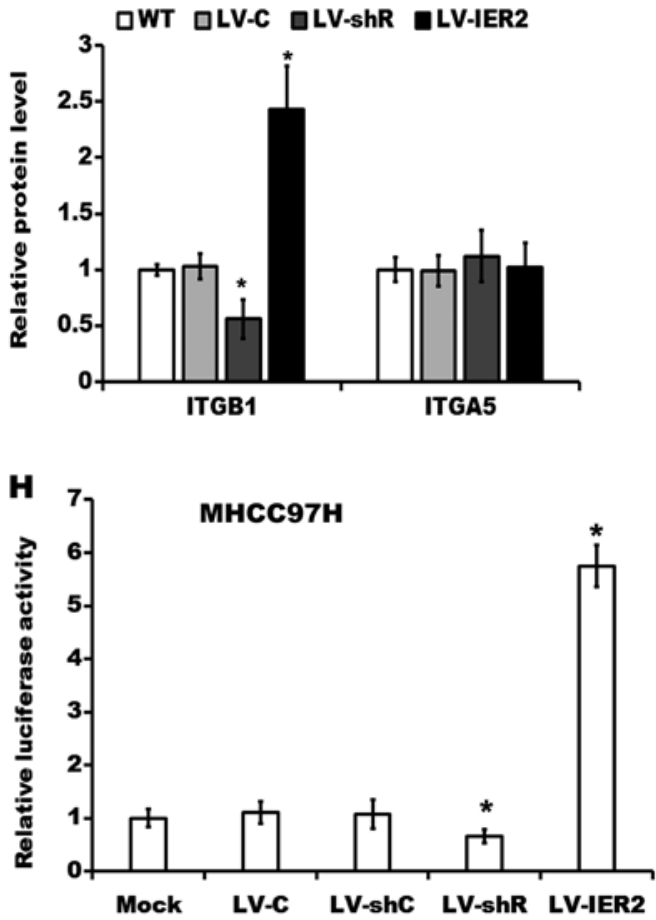

J MHCC97H
J

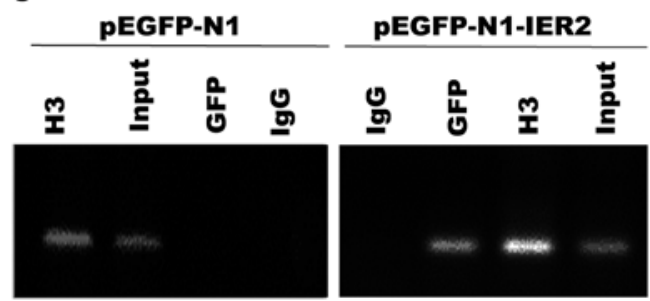

Figure 7. IER 2 promotes ITGB1 expression in HCC cells. (A and B) mRNA levels of ITGB1 and ITGA5 were measured from the indicated lentiviral-transduced (A) SMMC-7721 and (B) MHCC97H cells using RT-qPCR, and normalized against GAPDH. Data are the mean \pm SD ( $n=5$ ). "p<0.05 vs. the WT. (C-F) Protein levels of ITGB1 and ITGA5 were determined by western blot, normalized to GAPDH. Data are the mean \pm SD $(n=3)$. " $p<0.05$ vs. the WT. $(\mathrm{G}$ and $\mathrm{H})$ Luciferase reporter gene assays were performed in the indicated lentiviral-transduced $(\mathrm{G}) \mathrm{SMMC}-7721$ or $(\mathrm{H}) \mathrm{MHCC} 97 \mathrm{H}$ cells co-transfected with the luciferase reporter constructs and pTK-Renilla vectors, respectively. After $48 \mathrm{~h}$, the luciferase activity was determined. Data are the mean \pm SD from four independent experiments. " $\mathrm{p}<0.05$ vs. the WT. (I and J) ChIP assay was performed in (I) SMMC-7721 and (J) MHCC97H cells stably transfected with pEGFPN1-IER2 or pEGFP-N1 by using a GFP antibody, a positive control histone H3 antibody or a negative control rabbit IgG for immunoprecipitation followed by semi-quantitative PCR with human ITGB1 promoter-specific primers. Representative results are from one of three independent experiments. 
A
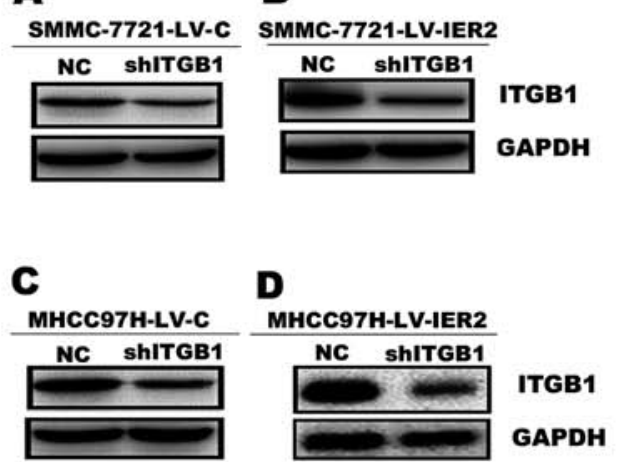

$\mathbf{E}$
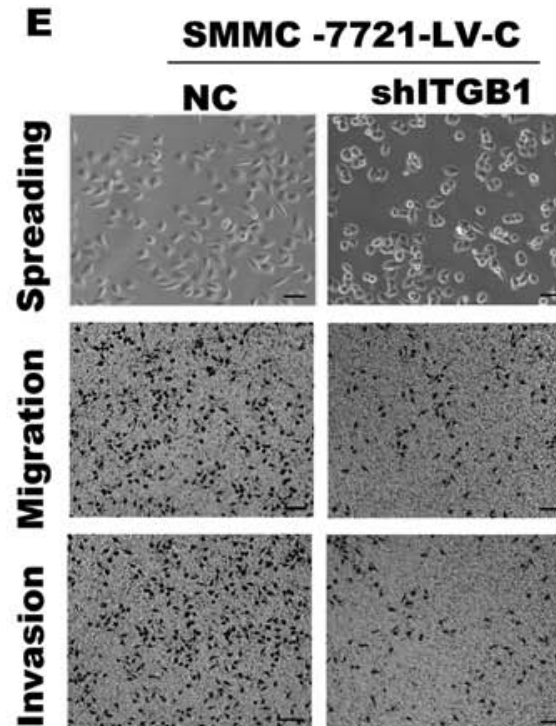

\section{6}

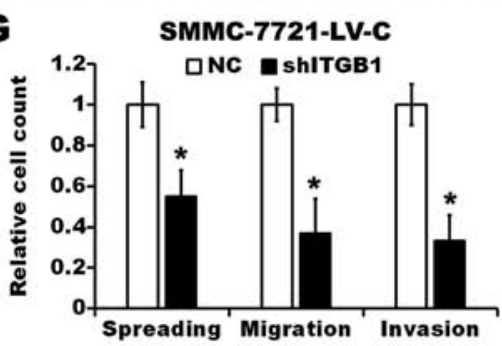

F SMMC-7721-LV-IER2

NC

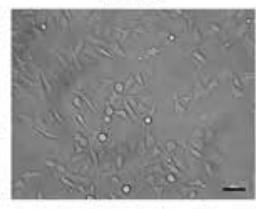
shITGB1
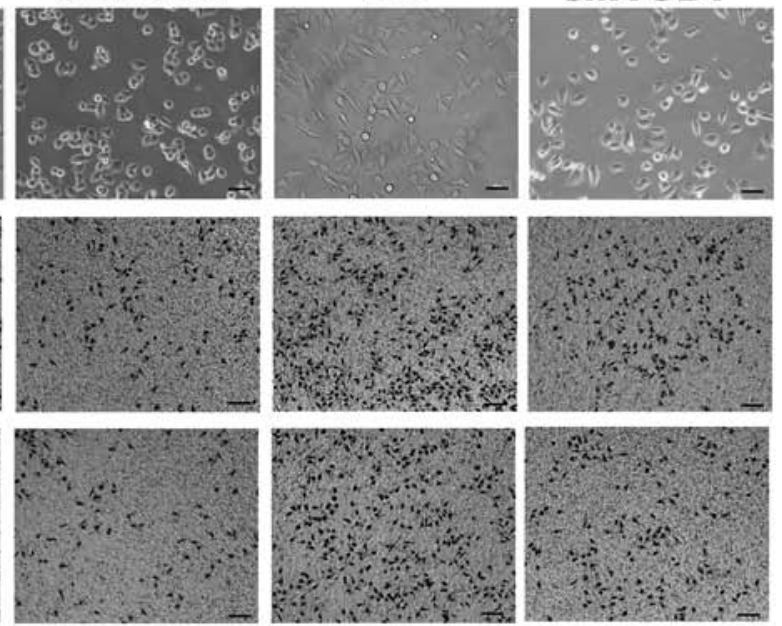

H

SMMC-7721-LV-IER2

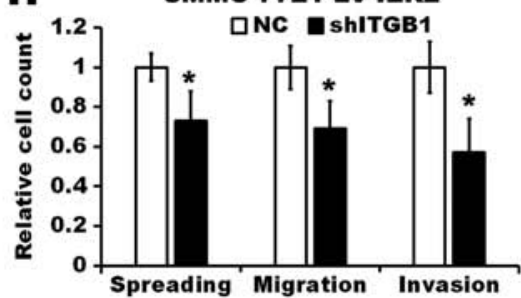

I MHCC97H-LV-C

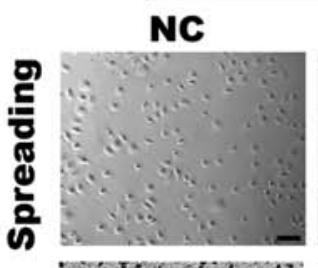
ShITGB1
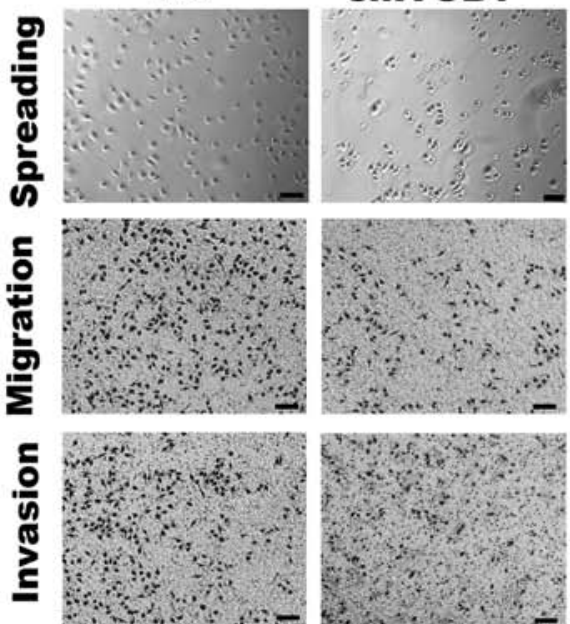

$\mathbf{K}$

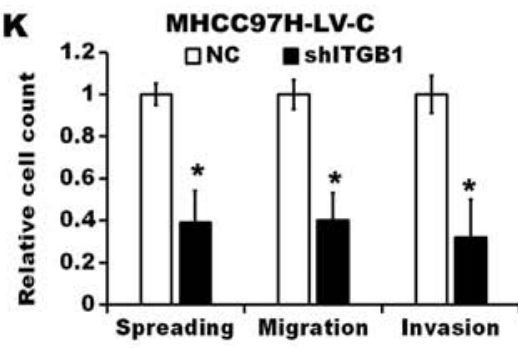

J MHCC97H-LV-IER2

NC ShITGB1
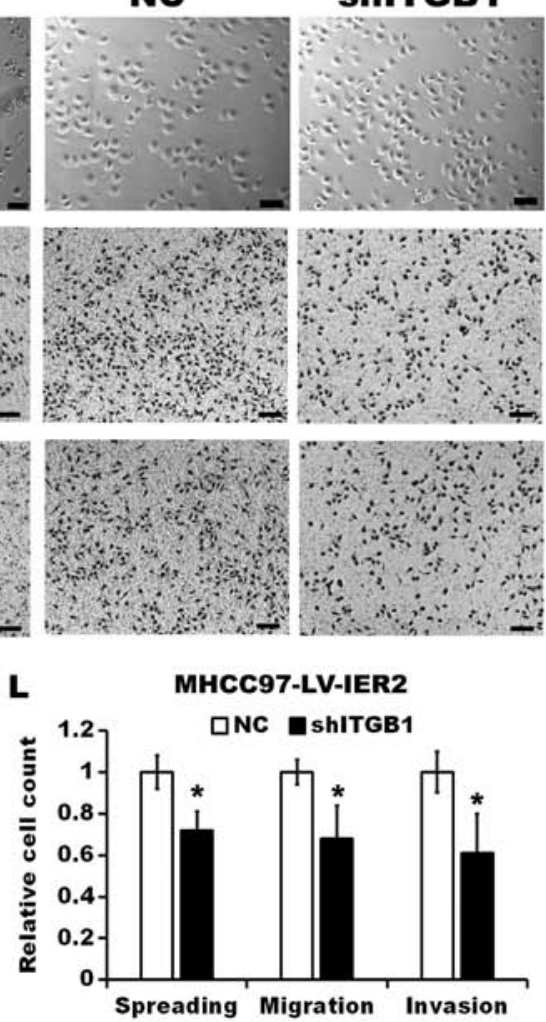

Figure 8. Effects of ITGB1 reduction on IER2-mediated cell spreading and motility. (A-D) Western blot analysis of ITGB1 in stably LV-C-transduced SMMC-7721 (SMMC-7721-LV-C) and MHCC97H (MHCC97H-LV-C) cells or the LV-IER2-transduced SMMC-7721 (SMMC-7721-LV-IER2) and MHCC97H (MHCC97H-LV-IER2) cells after transfected with shRNA vectors against ITGB1 (shITGB1) or with non-targeting shRNA vectors (NC) (n=3). (E, F, I and J) The indicated cells were allowed to spread on fibronectin-coated plates for 120 min or subjected to Transwell migration and invasion assays. The images represent cells from three independent experiments. Scale bars, $100 \mu \mathrm{m}$. (G, H, K and L) Quantification of cell spreading, migration and invasion is shown. Data are presented as mean $\pm \mathrm{SD}$ of three independent experiments. ${ }^{*} \mathrm{p}<0.05$ vs. the indicated $\mathrm{NC}$ 
A SMMC-7721

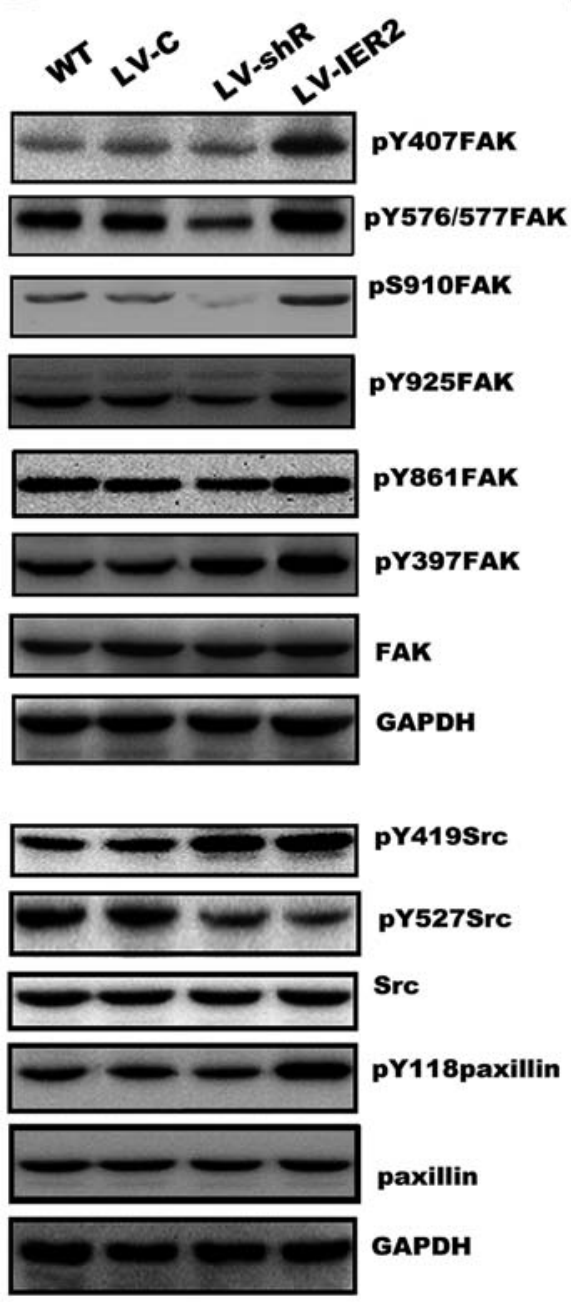

В Мнсс97н
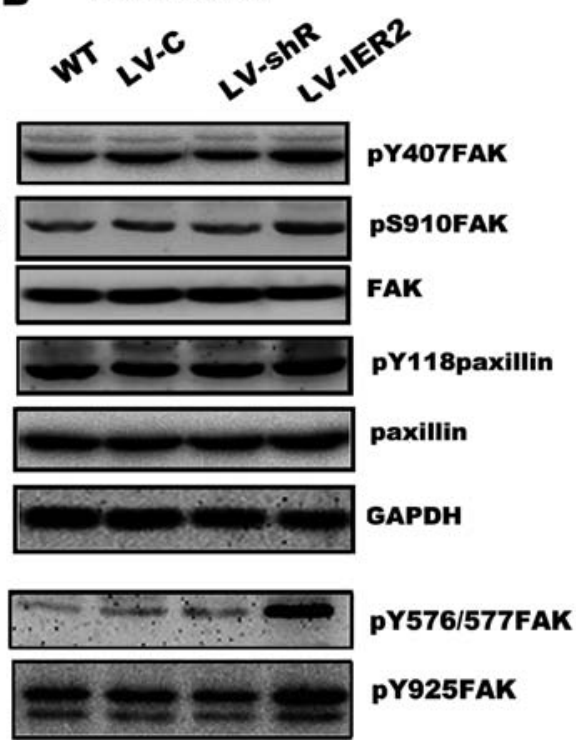

pY576/577FAK

PY925FAK

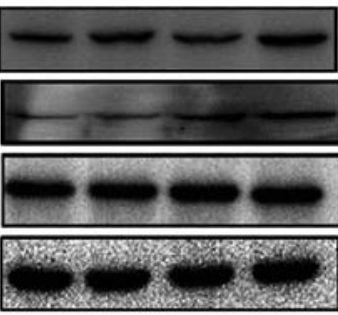

pY861FAK

pY397FAK

FAK

GAPDH

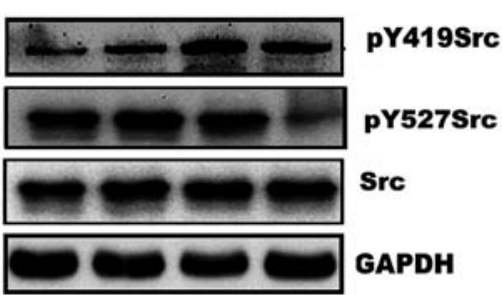

Figure 9. Effect of IER2 on expression of FAK, Src and paxillin in HCC cells. (A and B) Protein levels of FAK, Src and paxillin were analyzed from the indicated lentiviral-transduced (A) SMMC-7721 and (B) MHCC97H cells by western blotting with the corresponding antibodies, and GAPDH was the loading control $(n=3)$.

with high metastatic potential, and identified IER2 as a regulator of cell-fibronectin adhesion, spreading and motility of these two cell lines. We further defined a new pathway that involves the cell-ECM adhesion molecule ITGB1, and demonstrated the novel IER2-ITGB1 signaling critically contributed to the HCC cell-ECM adhesion and motility.

In this study, we first found that IER2 overexpression obviously induced cell migration and invasion, while silencing of IER2 decreased the motility of both SMMC-7721 and MHCC97H cells, suggesting that IER2 may function as an important regulator in HCC cell migration and invasion. Since cell motility requires cell-ECM adhesion, spreading and then invading the ECM $(13-15,25)$ we next evaluated whether IER2 induced HCC cell migration and invasion via its regulation for the cell-ECM adhesion and spreading. We demonstrated that IER2 overexpression promoted SMMC-7721 or MHCC97H cell adhesion and spreading onto the fibronectin (but not collagen type I or Matrigel), whereas IER2 knockdown repressed cell adhesion and spreading onto the fibronectin. Taken together, these findings indicated that IER 2 is an important regulator of cell spreading and adhesion onto the fibronectin, and promotion of the cell motility by IER 2 overexpression may be due to the increase in cell-fibronectin adhesion and spreading, whereas the decrease in the cell motility caused by IER 2 depression is probably attributed to the decreasing cell-fibronectin adhesion and spreading, and the endogenous IER2 expression is required for cell motility and adhesion.

Integrins are the major ECM receptors that consist of $\alpha$ and $\beta$ subunits and play very important roles in cell motility and tumor progression, especially in tumor invasion and metastasis (16-18,26). Among integrins, the heterodimers of ITGA5 and ITGB1, receptors for fibronectin, are thought to mediate tumor cell adhesion to fibronectin and closely correlate with cancer progression and metastasis (18). In the present study, we demonstrated that IER2 expression was positively correlated with the metastatic potential and associated with ITGB1 expression levels in HCC cell lines. These results prompted us to examine whether IER2 may regulate the expression of ITGA5 and ITGB1. We showed that IER2 expression enhanced ITGB1 expression both in mRNA and protein levels in SMMC-7721 and MHCC97H cells, while knockdown of IER2 reduced ITGB1 expression. Abundant expression of ITGA5 

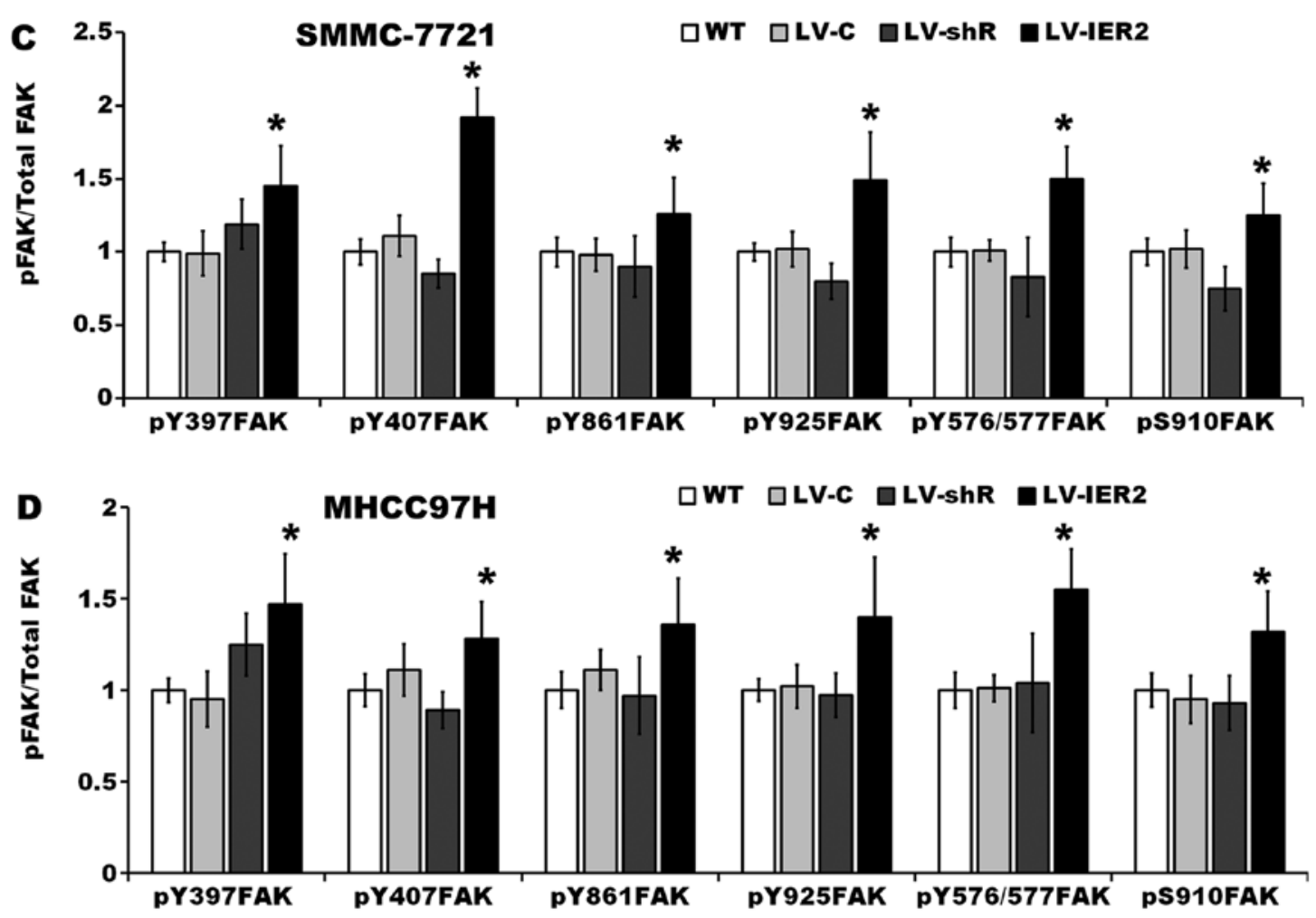
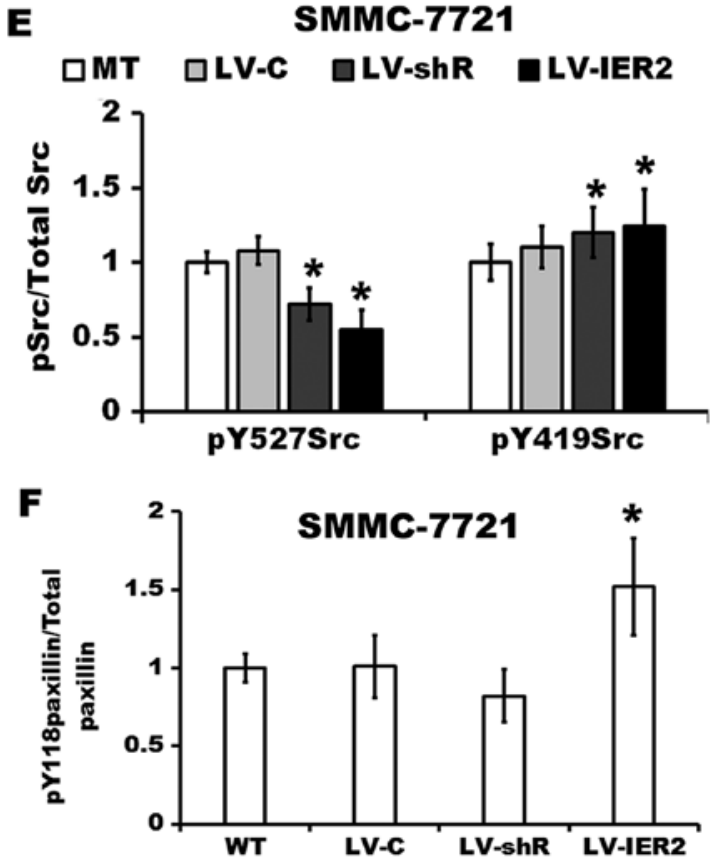

MHCC97H
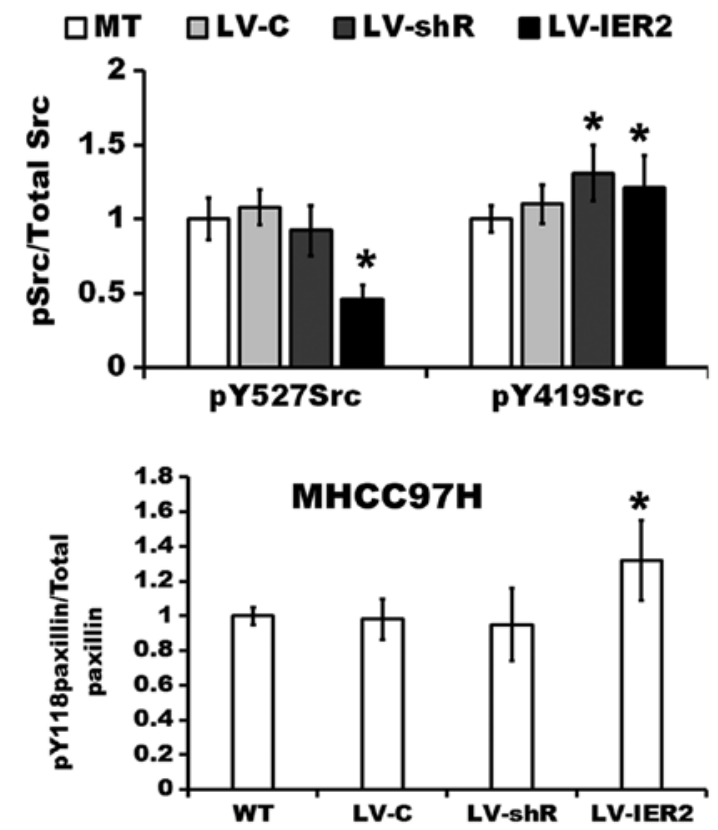

Figure 9. Continued. (C-F) Quantification of the band intensity was performed with ImageJ software, and expressed as fold-change of the WT after being normalized against GAPDH. Data are the mean \pm SD of three independent experiments. " $p<0.05$ vs. the WT.

was observed in HCC cell lines, but neither IER2 overexpression nor knockdown altered the expression of ITGA5 either in mRNA or protein levels in SMMC-7721 and MHCC97H cells. We also demonstrated that IER2 may regulate expression of ITGB1 in HCC cell lines by functioning as a potential transcriptional factor or transcriptional co-activator for ITGB1. Furthermore, we silenced ITGB1 in both SMMC-7721 and MHCC 97H cells stably transduced with LV-C or LV-IER2, and found that the increase of cell migration, invasion, and spreading on fibronectin in IER2 overexpressing cells was prevented by knockdown of ITGB1. Collectively, these findings suggested that IER2-induced cell-fibronectin adhesion, spreading and motility of the HCC cells may be mediated, at least in part, by promotion of ITGB1.

It is well documented that ITGB1 is the classic fibronectin receptor and mediates cell adhesion onto the ECM and confers higher metastatic capacity to a number of cancer cells (27-29). Upon integrin-mediated signaling activation, the heterodimers of ITGA5 and ITGB1 link fibronectin to induce phosphorylation of the FAK at Y397 and formation of the active FAK/Src 
complex, followed by further phosphorylation at other sites on the FAK and other downstream signaling molecules including paxillin, and subsequently modulating cell motility and cell-fibronectin adhesion $(24,30,31)$. Therefore, we further examined the role of IER2 in the integrin-mediated signaling pathway. We found that the FAK phosphorylation (at Y397, Y407, Y576/Y577, Y861, Y925 and S910), Src phosphorylation (at Y419), and paxillin phosphorylation were significantly upregulated, and the Src phosphorylation at Y527 [the inhibitory site for Src activation (32)] was downregulated in IER2 overexpressing HCC cells, suggesting that IER2 overexpression promoted HCC cell-fibronectin adhesion, spreading and motility by activating FAK, Src and paxillin via ITGB1. Unexpectedly, we did not find obvious opposite effects in IER2 knockdown cells. We found that silencing of IER2 resulted in only a slight, but no significant alteration of phosphorylation of FAK and paxillin, but also significant decrease of pY527Src and increase of pY419Src, suggesting that IER2 may have some uncharacterized functions or potential partners to regulate HCC cell motility and adhesion, and that IER2 activated ITGB1-mediated signal pathway is likely a part of a mechanism that participates in modulation of cell motility and adhesion of HCC cells. Collectively, these findings indicated that IER2-mediated ITGB1 expression and activation of ITGB1-FAK-Src-paxillin signal pathway was likely, at least in part, responsible for IER2-promoted cell-ECM adhesion and motility of the HCC cells, and studies to identify potential partners and the detail mechanisms of IER2 in regulating HCC cell-ECM adhesion and motility are under way.

In conclusion, we demonstrated a novel pathway modulating HCC cell motility and cell-ECM adhesion by IER2, and identified that IER 2 expression is positively correlated with the metastatic potential of HCC cell lines, and that IER 2 promoted HCC cell motility and the cell-fibronectin adhesion as the result of the activation of the ITGB1-FAK-Src-paxillin signal pathway. Further study of the underlying mechanism as to how IER2 depletion coordinates the ITGB1-FAK-Src-paxillin signaling is ongoing, however, the findings presented here may reinforce a role for IER2 as a novel molecular target for HCC.

\section{Acknowledgements}

This study was supported by the National Nature Science Foundation of China (no. 81172278).

\section{References}

1. Shimizu N, Ohta M, Fujiwara C, Sagara J, Mochizuki N, Oda $\mathrm{T}$ and Utiyama $\mathrm{H}$ : Expression of a novel immediate early gene during 12-O-tetradecanoylphorbol-13-acetate-induced macrophagic differentiation of HL-60 cells. J Biol Chem 266: 12157-12161, 1991.

2. Zeng F, Hon CC, Sit WH, Chow KY, Hui RK, Law IK, Ng VW, Yang XT, Leung FC and Wan JM: Molecular characterization of Coriolus versicolor PSP-induced apoptosis in human promyelotic leukemic HL-60 cells using cDNA microarray. Int J Oncol 27: 513-523, 2005

3. Takaya T, Kasatani K, Noguchi S and Nikawa J: Functional analyses of immediate early gene ETR 101 expressed in yeast. Biosci Biotechnol Biochem 73: 1653-1660, 2009.

4. Chen L, Ma S, Li B, Fink T, Zachar V, Takahashi M, Cuttichia J, Tsui LC, Ebbesen P and Liu X: Transcriptional activation of immediate-early gene ETR101 by human T-cell leukaemia virus type I Tax. J Gen Virol 84: 3203-3214, 2003.
5. Hong SK and Dawid IB: FGF-dependent left-right asymmetry patterning in zebrafish is mediated by Ier2 and Fibp1. Proc Natl Acad Sci USA 106: 2230-2235, 2009.

6. Neeb A, Wallbaum S, Novac N, Dukovic-Schulze S, Scholl I, Schreiber C, Schlag P, Moll J, Stein U and Sleeman JP: The immediate early gene Ier 2 promotes tumor cell motility and metastasis, and predicts poor survival of colorectal cancer patients. Oncogene 31: 3796-3806, 2012.

7. Wu W, Zhang X, Lv H, Liao Y, Zhang W, Cheng H, Deng Z, Shen J, Yuan Q, Zhang Y, et al: Identification of immediate early response protein 2 as a regulator of angiogenesis through the modulation of endothelial cell motility and adhesion. Int J Mol Med 36: 1104-1110, 2015.

8. Wu W, Zhang X, Liao Y, Zhang W, Cheng H, Deng Z, Shen J, Yuan Q, Zhang Y and Shen W: miR-30c negatively regulates the migration and invasion by targeting the immediate early response protein 2 in SMMC-7721 and HepG2 cells. Am J Cancer Res 5: 1435-1446, 2015.

9. Yan M, Li H, Zhu M, Zhao F, Zhang L, Chen T, Jiang G, Xie H, Cui Y, Yao M, et al: G protein-coupled receptor 87 (GPR87) promotes the growth and metastasis of $\mathrm{CD}_{133^{+}}$cancer stem-like cells in hepatocellular carcinoma. PLoS One 8: e61056, 2013.

10. Woodhouse EC, Chuaqui RF and Liotta LA: General mechanisms of metastasis. Cancer 80 (Suppl): 1529-1537, 1997.

11. Geiger TR and Peeper DS: Metastasis mechanisms. Biochim Biophys Acta 1796: 293-308, 2009.

12. Wu W, Zhang X, Qin H, Peng W, Xue Q, Lv H, Zhang H, Qiu Y, Cheng H, Zhang Y, et al: Modulation of tumor cell migration, invasion and cell-matrix adhesion by human monopolar spindleone-binder 2. Oncol Rep 33: 2495-2503, 2015.

13. Khalili AA and Ahmad MR: A review of cell adhesion studies for biomedical and biological applications. Int J Mol Sci 16: 18149-18184, 2015.

14. Ishikawa $\mathrm{T}$ and Kramer RH: Sdc1 negatively modulates carcinoma cell motility and invasion. Exp Cell Res 316: 951-965, 2010.

15. Desgrosellier JS and Cheresh DA: Integrins in cancer: Biological implications and therapeutic opportunities. Nat Rev Cancer 10: 9-22, 2010

16. Margadant C, Monsuur HN, Norman JC and Sonnenberg A: Mechanisms of integrin activation and trafficking. Curr Opin Cell Biol 23: 607-614, 2011.

17. Keely S, Glover LE, MacManus CF, Campbell EL, Scully MM, Furuta GT and Colgan SP: Selective induction of integrin beta1 by hypoxia-inducible factor: Implications for wound healing. FASEB J 23: 1338-1346, 2009.

18. Qin L, Chen X, Wu Y, Feng Z, He T, Wang L, Liao L and Xu J: Steroid receptor coactivator-1 upregulates integrin $\alpha_{5}$ expression to promote breast cancer cell adhesion and migration. Cancer Res 71: 1742-1751, 2011.

19. Zhou L, Wang DS, Li QJ, Sun W, Zhang Y and Dou KF: The downregulation of Notch1 inhibits the invasion and migration of hepatocellular carcinoma cells by inactivating the cyclooxygenase-2/Snail/E-cadherin pathway in vitro. Dig Dis Sci 58: $1016-1025,2013$.

20. Wang C, Jin G, Jin H, Wang N, Luo Q, Zhang Y, Gao D, Jiang K, Gu D, Shen Q, et al: Clusterin facilitates metastasis by EIF3I/Akt/MMP13 signaling in hepatocellular carcinoma. Oncotarget 6: 2903-2916, 2015.

21. Wang C, Jiang K, Kang X, Gao D, Sun C, Li Y, Sun L, Zhang S, Liu X, Wu W, et al: Tumor-derived secretory clusterin induces epithelial-mesenchymal transition and facilitates hepatocellular carcinoma metastasis. Int J Biochem Cell Biol 44: 2308-2320, 2012.

22. Fraley SI, Feng Y, Krishnamurthy R, Kim DH, Celedon A, Longmore GD and Wirtz D: A distinctive role for focal adhesion proteins in three-dimensional cell motility. Nat Cell Biol 12: 598-604, 2010.

23. van Nimwegen MJ and van de Water B: Focal adhesion kinase: A potential target in cancer therapy. Biochem Pharmacol 73: 597-609, 2007.

24. Mitra SK and Schlaepfer DD: Integrin-regulated FAK-Src signaling in normal and cancer cells. Curr Opin Cell Biol 18: 516-523, 2006.

25. Webb DJ, Donais K, Whitmore LA, Thomas SM, Turner CE, Parsons JT and Horwitz AF: FAK-Src signalling through paxillin, ERK and MLCK regulates adhesion disassembly. Nat Cell Biol 6: 154-161, 2004.

26. Hood JD and Cheresh DA: Role of integrins in cell invasion and migration. Nat Rev Cancer 2: 91-100, 2002. 
27. Xu JK, Chen HJ, Li XD, Huang ZL, Xu H, Yang HL and Hu J: Optimal intensity shock wave promotes the adhesion and migration of rat osteoblasts via integrin $\beta 1$-mediated expression of phosphorylated focal adhesion kinase. J Biol Chem 287: 26200-26212, 2012.

28. Moursi AM, Globus RK and Damsky CH: Interactions between integrin receptors and fibronectin are required for calvarial osteoblast differentiation in vitro. J Cell Sci 110: 2187-2196, 1997.

29. Broustas CG,Zhu A and Lieberman HB: Rad9 protein contributes to prostate tumor progression by promoting cell migration and anoikis resistance. J Biol Chem 287: 41324-41333, 2012.

30. Carvajal-Gonzalez JM, Mulero-Navarro S, Roman AC, Sauzeau V, Merino JM, Bustelo XR and Fernandez-Salguero PM: The dioxin receptor regulates the constitutive expression of the vav3 proto-oncogene and modulates cell shape and adhesion. Mol Biol Cell 20: 1715-1727, 2009.
31. Mulero-Navarro S, Pozo-Guisado E, Pérez-Mancera PA, Alvarez-Barrientos A, Catalina-Fernández I, Hernández-Nieto E, Sáenz-Santamaria J, Martínez N, Rojas JM, Sánchez-García I, et al: Immortalized mouse mammary fibroblasts lacking dioxin receptor have impaired tumorigenicity in a subcutaneous mouse xenograft model. J Biol Chem 280: 28731-28741, 2005.

32. Crosara-Alberto DP, Inoue RY and Costa CR: FAK signalling mediates NF-kappaB activation by mechanical stress in cardiac myocytes. Clin Chim Acta 403: 81-86, 2009. 